\title{
Platelet cytochrome c-oxidase activity in patients with acute schizophrenia in the course of their treatment with risperidone
}

\author{
Gulnur Sh. Burbaeva ${ }^{1}$, Irina S. Boksha ${ }^{1}$, Marina S. Turishcheva ${ }^{1}$, Olga K. Savushkina ${ }^{1}$, \\ Alan G. Beniashvili², Georgiy E. Rupchev ${ }^{2}$, Margarita A. Morozova ${ }^{2}$ \\ ${ }^{1}$ Laboratory of Neurochemistry, Mental Health Research Centre, Russian Academy of Medical Sciences, Moscow, Russia; \\ *Corresponding Author: neurochem06@mail.ru \\ ${ }^{2}$ Laboratory of Psychopharmacology at the Clinical Department Mental Health Research Centre, Russian Academy of Medical Sci- \\ ences, Moscow, Russia; margmorozova@gmail.com
}

Received 5 October 2011; revised 15 October 2011; accepted 19 October 2011

\begin{abstract}
Objectives: Testing a hypothesis, that platelet cytochrome c-oxidase (COX) activities in patients with paranoid schizophrenia, acute episode, may be linked to dynamics of their clinical patterns and quality of cognitive functioning under antipsychotic treatment. Methods: Psychopathological (PANSS, NSA-16) and cognitive assessments; platelet COX enzymatic activity determination, post-hoc nonparametric statistical analysis. Results: Psychopathological and cognitive assessments were done and blood was sampled in patients before (at baseline) and after treatment with risperidone. Following regularities were found after the treatment of patients: Significant elevation of COX, wherein the higher was COX at baseline, the more prominent was decrease in PANSSneg and NSA rates; significant negative correlation between COX and executive time in cognitive tests. When the patient group was divided by median of COX at baseline into two subgroups (greater or equal median, and <median), significantly larger amount of patients with PANSSpos decrease by $>\mathbf{2 0} \%$ was assigned to the first subgroup; significantly larger amount of patients with PANSSneg by $<20 \%$ was assigned to the second group. Conclusions: Therapy with risperidone seems to be more effective for patients with higher COX activity at baseline, but this fact requires further study.
\end{abstract}

Keywords: Schizophrenia; Risperidone; Biochemical Markers; Acute Phase Treatment; Atypical Antipsychotics

\section{INTRODUCTION}

Many evidences have been obtained for energy metabolism impairment in brain of patients with mental disorders [1-6]. The decrease in rates of glucose oxidation and oxygen consumption in some brain areas of demented patients with Alzheimer's disease [1,2] or schizophrenia [4,7] has been demonstrated by MRT and PET methods. Significant decrease in cytochrome $c$ oxidase activity (COX - the complex IV of mitochondrial respiratory chain) has been observed in several autopsied brain structures obtained from patients with schizophrenia as compared with control $[4,8]$. This fact suggests for defect of oxidation phosphorylation in brain of patients with schizophrenia, putatively resulting in energy metabolism impairment [9].

Blood platelets are often used for biochemical modeling of processes occurring in nervous tissue, wherein platelet mitochondria possess the complete respiratory electron transport chain, including all its enzymatic complexes, sufficiently active for in vitro biochemical studies with clinical aims [10,12,13]. Attempts have been made to evaluate platelet COX activity in mental disorders, such as dementia (due to Alzheimer's disease), and mild cognitive impairment (MCI) [14-16], cognitive decline in Parkinson disease [17], and schizophrenia. Platelet COX activity in the groups with dementia and MCI was found lower than that in controls, wherein the patients with MCI "occupied" positions between the control group and demented patients [14-16]. Taken together this data has enabled to put forward an assumption on probable diagnostic validity of $\mathrm{COX}$ as a biological marker of Alzheimer's disease [14]. Another group of investigators explored the diagnostic aspect of platelet respiratory chain activity and found elevation of respiratory complex I activity in patients with schizophrenia, 
whereas no difference was found in COX activity between the samples from patients with schizophrenia and controls [13].

In the present study we tested a hypothesis, which assumed that platelet COX activities in patients with acute schizophrenia might be linked to the dynamics of their clinical patterns and quality of their cognitive functioning under the antipsychotic treatment. The study included following tasks: comparative estimation of COX activity in patients with schizophrenia and in control group; estimation of COX activity changes under antipsychotic treatment; evaluation of links between COX and clinical and neurocognitive assessments before treatment course ("at baseline”) and after the treatment. Besides, on the basis of the tasks solved, we attempted to evaluate a possibility of COX usage for prognosis of antipsychotic treatment efficacy.

\section{MATERIALS AND METHODS}

\subsection{Subjects and Methods of the Study}

After receiving approval for the study from the Ethics Committee of MHRC RAMS, 27 patients (men, 20-56 years old; median 33 years, 25\%-, and 75\%-quartiles are 25 and 44 years, respectively) with acute schizophrenia, paranoid type (DSM-IV 295.30), have been included into the study and assigned to the antipsychotic treatment with risperidone (4-6 mg per day).

Psychopathological assessments by PANSS, negative symptom scale NSA-16, and neurocognitive assessments (battery of neurocognitive tests evaluating various aspects of memory, attention, executive functioning) were regarded at baseline and after treatment course with risperidone for 2 months, blood was sampled at the same time ranges for COX activity assay.

Neurocognitive assessment tests:

1) Working memory: Wechsler memory scale, subtest $\mathrm{V}$ : series A; series B; sum A and B.

2) Associative memory: Wechsler memory scale, subtest VII;

3) Psychomotor speed: WAIS test, subtest VII: Symbol coding.

4) Verbal memory: Text No1 reconstruction, and Text No2 reconstruction.

5) Visual-spatial memory: Benton visual intention test (BVRT), Fifth Education.

6) Attention: Schulte tables and Bourdon test (pencil-and-paper test), Continues attention task (CAT), (Computerised testing).

7) Executive functions: Tower of London (TOL dx); Computerised Wisconsin Card Sort Task Version 4 (WCST).
The control group consisted of 25 volunteers (men, 17-43 years old; median 25 years, 25\%- and 75\%-quartiles are 20 , and 27 years, respectively) - healthy persons with no history of mental or neurological disorders.

There was found significant age difference between the patient and control groups ( $p<0.00033$ ), however, no influences of age on COX activity in controls or patients were found (Spearmen $\mathrm{R}<0.22$, $\mathrm{p}>0.32$ ), therefore we admitted this control group.

Isolation of platelets and extraction method for COX activity determination.

Each blood sample was processed in 2 hours after sampling. Blood plasma prepared from $10 \mathrm{ml}$ of venous blood taken with citrate buffer containing dextrose, $\mathrm{pH}$ $5.7(1: 5, \mathrm{v} / \mathrm{v})$, by centrifugation at $1000 \mathrm{rpm}$ for $10 \mathrm{~min}$ at room temperature (Beckman J-6 centrifuge), was carefully collected for following centrifugation at 10,000 rpm for $20 \mathrm{~min}$ at $5^{\circ} \mathrm{C}$ (Beckman J-21 centrifuge, JA20.1 rotor). The supernatant was removed, and the pellet was washed with the citrate buffer by centrifugation, supernatant removed, and the pellet re-suspended in $62.5 \mathrm{mM}$ Tris-HCl buffer, $\mathrm{pH}$ 6.8, then frozen in liquid nitrogen and stored at $-70^{\circ} \mathrm{C}$ prior the analysis of COX activity. Just before the measurement the sample was defrosted, and n-dodecyl-beta-D-maltoside was added to its final concentration of $1 \%$, then incubated for $10 \mathrm{~min}$ at $4^{\circ} \mathrm{C}$, centrifuged (10 $\min 10,000 \mathrm{~g}$ at $4^{\circ} \mathrm{C}$ ), and COX activity was measured in supernatant.

COX enzymatic activity was determined by kinetic method using spectrophotometer $(\lambda=550 \mathrm{~nm})[18,19]$. Specific activity of COX was calculated (U/mg), taken the total protein measured by Lowry et al. [20].

Internal standard

Platelet sample collection accumulated in the Laboratory of Neurochemistry RAMS includes the samples from healthy volunteers used for the internal standard preparation. Protein extracts prepared from these samples according with the described above procedure are used as the internal standard (a gauge) in comparative COX estimations in each experiment.

\subsection{Statistical Analysis}

Clinical data, demography, and biochemical data of the patients composed a database. Nonparametric statistics (Spearmen correlations, Wilcoxon Matched Pairs Test, Mann-Whitney U-test, Chi-square distribution with Yates correction factor using Statistica 6.0 software) was employed for assessment of changes in the target parameters and links between them.

\section{RESULTS}

1) Comparison of COX baseline activity in group of 
Table 1. Result of statistical COX activity data processing in patient group (before the treatment course) and in controls by Mann-Whitney U-Test.

\begin{tabular}{|c|c|c|c|c|c|c|c|}
\hline & Rank Sum & Rank Sum & $\mathrm{U}$ & Z & $\begin{array}{c}\text { Patients with schizophrenia } \\
\mathrm{N}\end{array}$ & $\begin{array}{c}\text { Controls } \\
\mathrm{N}\end{array}$ & p-level \\
\hline $\operatorname{cox}$ & 462 & 916 & 84 & -4.642 & 27 & 25 & 0.000001 \\
\hline
\end{tabular}

patients with schizophrenia (before the beginning of treatment course with risperidone) and in control group.

Comparison of COX baseline activity in group of patients with schizophrenia and in control group (by Mann-Whitney U-test) has revealed, that platelet COX activity is significantly decreased in the patient group ( $\mathrm{p}$ $=0.000001$, Table 1).

The distributions of observation numbers of various COX activities in the control group and in the group of patients before the treatment course are shown in the Figures 1(a) and (b). Comparison of these diagrams demonstrates, that the number of lower COX activity observations is higher in the patient group than in the control group.

2) COX comparison in patient group before and after treatment course.

Comparison of COX activity before and after the treatment course by non-parametric statistics (Wilcoxon Matched Pairs Test) has demonstrated its significant change (Table 2), wherein the increase in COX activity was observed in 20 patients, and its decrease was observed in 7 patients. Although COX has increased in most patients after the treatment course, the patient group has been found significantly different still from the control group ( $p=0.00015)$.

The increase in COX activity after the treatment course is illustrated with the figure showing diagrams of COX activity distribution in the patient group (COX activity vs. observations' number) before and after the treatment course (diagrams b and c, respectively). It is obviously from the comparison of the diagrams that the number of patients with higher COX activities increases after the treatment course.

3) Search for links between COX activity and alterations in clinical and cognition assessments under the treatment course with risperidone.

Since the decreased COX activity has been found in patients with schizophrenia in comparison with controls, we have supposed this parameter has to be reflecting the severity of psychosis and cognitive dysfunction in the patients. Search for links between COX activity and these assessments has provided no direct support for this hypothesis, however, baseline COX activity has proved to have prognostic value due to its link with PANSSneg magnitude of alteration under the treatment course $(\mathrm{R}=$ 0.45, $p<0.02$ ): the higher was baseline COX activity, the more prominent was the decrease in deficit rates.
Similar link was found with magnitude of NSA decrease after the treatment course $(\mathrm{R}=0.48, p<0.05)$.

The patients with decreasing COX activity after the treatment course $(\mathrm{N}=7)$ displayed negative significant correlations between COX activity and PANSS total and PANSS psychopathological scores at baselines $(\mathrm{R}=$ $-0.85, p<0.01, \mathrm{R}=-0.82, p<0.02$, respectively), i.e., the higher is COX at baseline, the less is psychotic symptoms' score.

The Figure 1(b) shows, that most patients demonstrate COX baseline activities near the median in the group (60 U/mg), however, prominent deviations from median value are seen as well. Hence, the group of patients was divided into two subgroups by COX baseline activity “ $\geq$ median" (subgroup 1 ) and " $<$ median” (subgroup 2) (no significant difference in age between them).

Significantly higher number of patients with decreasing PANSS positive by $20 \%$ and more has been detected in the subgroup 1, and the subgroup 2 contained signifycantly higher number of patients with PANSS negative score decreasing by less than $20 \%$ after the treatment $\left(\mathrm{X}^{2}\right.$ $=9.53$, Yates corrected $=7.6, p<0,01$, and $X^{2}=6.52$, Yates corrected $=4.53, p<0.025$, respectively). These findings mean, that patients with higher COX baseline activities demonstrate more prominent reduction of psychotic symptoms than those with lower COX baseline activities.

Patients with COX baseline activity $\geq$ median (subgroup 1) have demonstrated also significant improvement in results of neurocognitive testing: Psychomotor speed (Symbol coding), Total Executive Time of TOL, Middle Total Executive Time of TOL, and Psychomotor Speed.

No significant changes in results of clinical assessments were found in patients with COX baseline activity < median (subgroup 2), however, a significant improvement was observed in results of neurocognitive testing (Working memory and Verbal memory reconstruction).

The negative significant correlation was found in the total patient group between COX activity and time spent

Table 2. Result of comparison of COX activity in the patient group before (at baseline) and after the treatment course by Wilcoxon Matched Pairs Test.

\begin{tabular}{ccccc}
\hline & Valid & $\mathrm{T}$ & $\mathrm{Z}$ & $\mathrm{p}$-level \\
\hline $\begin{array}{c}\text { COX baseline \& COX after } \\
\text { the treatment }\end{array}$ & 27 & 65.500 & 2.414 & 0.016 \\
\hline
\end{tabular}




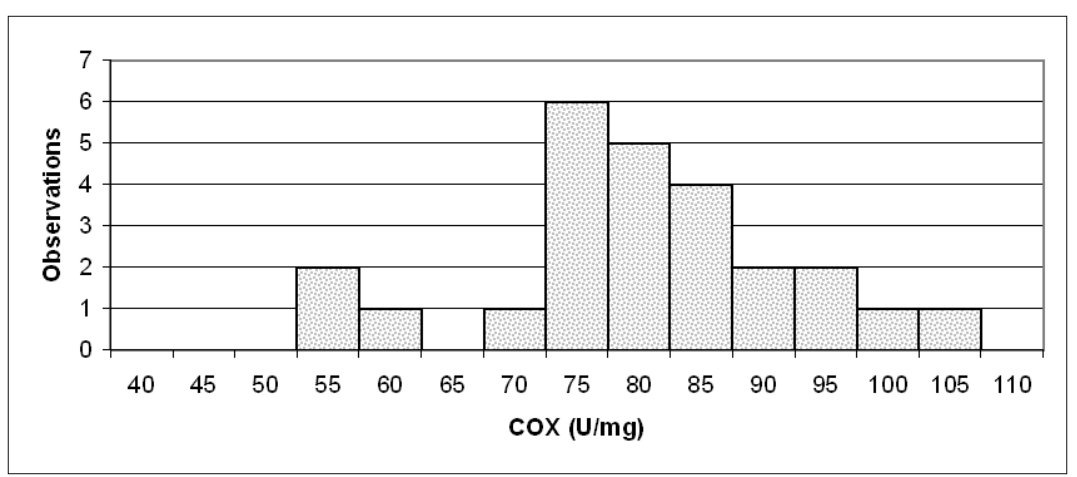

(a)

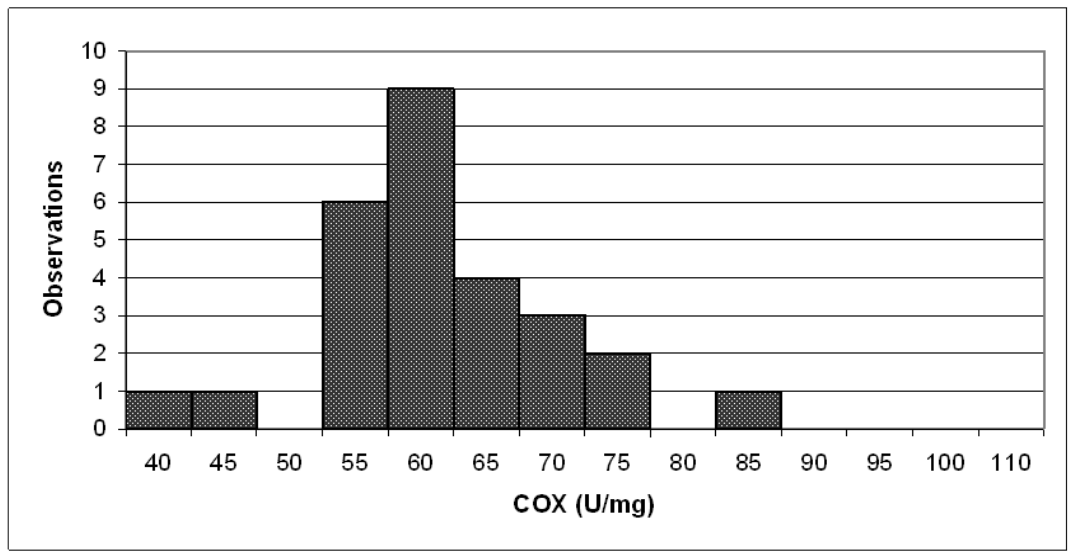

(b)

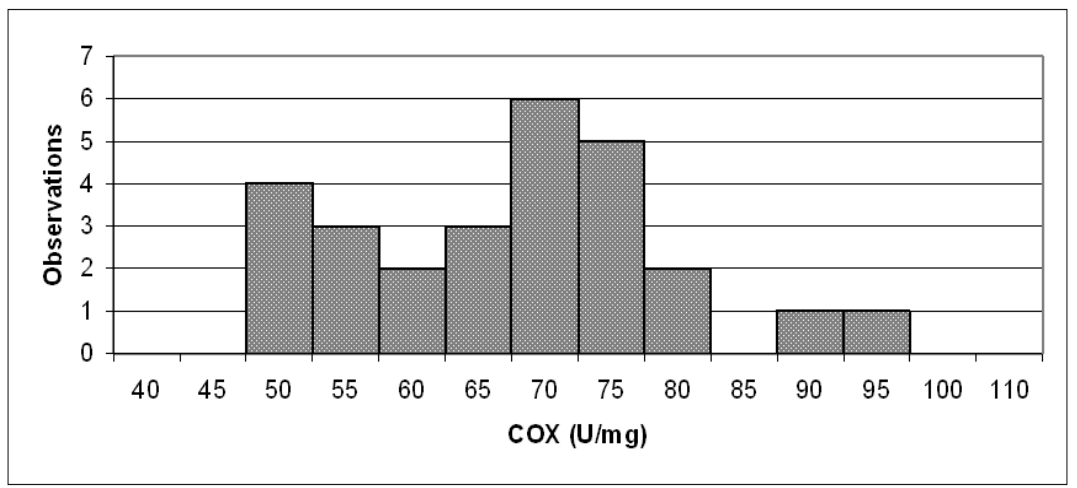

(c)

Figure 1. Observation numbers plotted versus various COX activities (U/mg) in control group (a), in patient group before treatment course (b), and in the patient group after the treatment course with risperidone (c).

for planning tests after the treatment course $(\mathrm{R}=-0.44$, $\mathrm{p}<0.03$ ). This link means that the higher is COX activity, the easier patient fulfills these tests after the treatment.

\section{CONCLUSIONS}

Platelet COX activity is significantly decreased in the studied group of patients with schizophrenia at baseline (before the antipsychotic treatment course) in comparison with control group.

Significantly higher number of patients with significantly better dynamics of clinical parameters is revealed in the subgroup of patients with COX baseline activities 
$\geq$ median. This is fairly for psychotic symptoms (more than $20 \%$ reduction of PANSS total score), and for mental deficit (significant reduction of NSA scores). Some results of neurocognitive tests have improved after the treatment therewith.

One would suppose, that the COX baseline levels $\geq$ median in acute state of patients with schizophrenia could be a sign of favorable dynamics of psychotic episode, and, possibly, positive response to antipsychotic treatment, although note, that the last statement requires an additional study.

\section{DISCUSSION}

In the present work, activity of enzymatic complex IV (cytochrome c-oxidase, COX) determined in platelet fraction enriched with mitochondrial membranes has proved to be sufficient for quantitative comparative studies in all inspected subjects. The platelet COX activity has been found therewith significantly decreased in the group of patients with paranoid schizophrenia (DSM -IV-TR 295.3), in comparison with control group. Review of Dr. Kato [21] pointed out some inconsistency in results obtained by different researchers, who studied enzymatic activity of mitochondrial electron transport chain and expression levels of genes encoding its complexes in schizophrenia on various populations and various tissues from patients. Recent data is not uniform as well $[13,22]$. So, significantly increased activity and gene expression of complex I (NADH: ubiquinone oxidoreductase) in blood of patients with schizophrenia has been reported [13], whereas decreased NDUFV2 expression levels have been detected in other study on patients with schizophrenia from Caucasian population [22].

The data indicating that the activity of complex I is higher in patients than in controls has enabled the authors [13] to propose complex I as diagnostic peripheral marker for schizophrenia. As for COX, the authors stated the absence of significant differences in its activity between patients and controls, but the experimental data on individual COX activity was not published making impossible a comparison between these results and data of the present work. However, one could suppose from our data, that "normalizing" platelet enzymatic activities by COX (proposed by Ben-Shachar and Klein) would result in overestimations of these activities in patients with schizophrenia.

Some disagreement in data on influence of neuroleptics on the activity of respiratory complexes is seen in literature as well, as noted earlier by Kato [21]. The paper of Ben-Shachar and Klein [13] reports on higher activity of complex I in patients treated with neuroleptics versus untreated patients. Other research groups reported on inhibition of complex I activity with neuroleptics (more prominent with typical, such as haloperidol, chlorpromazine, less prominent with atypical, such as risperidone), whereas COX activity was either not influenced, or affected. These results were obtained in vitro [23], in model experiments with animals [24], and on mononuclears in peripheral blood of patients with schizophrenia [25].

In the present study, significant elevation of COX activity was observed in the most patients after treatment course with risperidone, possibly it is associated with improvement of their clinical state. So, our special attention was paid to the treatment efficiency (clinical response) evaluation in the patients. In our previous study, when searching for biochemical predictor of pharmacological therapy efficiency we have taken the decrease in PANSS total for positive treatment effect [26]. In the present work, we additionally regard PANSS positive, PANSS negative, PANSS psychopathological, NSA, and neurocognitive tests.

The initially higher COX activity has been found to account for favorable prognosis in respect to whole clinical dynamics' pattern, especially to psychotic symptoms. This may be an indication, that higher level of energy metabolism is a good "background" for improvement in mental state of patients with schizophrenia, and, vice versa, lower level-a bad one. The initially higher COX activities ( $\geq$ median in total group) are associated with favorable prognosis for improvement of cognitive test results (WAIS subtest 7). Taking into account that this sub-test is the most complex by the number of intellectual functions included and is associated with various properties of attention, perception, visual- motor coordination, rate of new skills' formation, and ability of visual-motor stimuli integration [27], the successful improvement in the results of this test can be considered as a sign of cognitive function enhancement.

We recognize that the design of the present study does not allow unambiguous stating, that favorable clinical dynamics is associated with the therapy employed only, but we surely do admit this, taking into account numerous risperidone studies supporting its high antipsychotic activity [28]. Hence, one can admit, that the found link between the baseline COX activity and positive response to the treatment of patients in acute state is not casual: possibly, these patients have yet a "potential" to restore, whereas the patients with low COX level possibly need in additional therapy supporting energy metabolism.

Thus, the measurement of single parameter, such as platelet COX activity, related to platelet mitochondrial respiratory chain could have some prognostic value for prediction of individual efficacy of medication with risperidone in patients with schizophrenia (although, obviously, inspection of larger groups of patients is neces- 
sary for accomplishment individual prognosis, and an additional study is needed on dependence of COX activity on various factors).

\section{REFERENCES}

[1] Mohanakrishnan, P., Fowler, A.H., Vonsattel, J.P., Husain, M.M., Jolles, P.R., Liem, P. and Komoroski, R.A. (1995) An in vitro ${ }^{1} \mathrm{H}$ nuclear magnetic resonance study of the tempoparietal cortex of Alzheimer brains. Experimental Brain Research, 102, 503-510.

doi:10.1007/BF00230654

[2] Kennedy, A.M., Frackowiak, R.S., Newman, S.K., Bloomfield, P.M., Seaward, J., Roques, P., Lewington, G., Cunningham, V.J. and Rossor, M.N. (1995) Deficits in cerebral glucose metabolism demonstrated by positron emission tomography in individuals at risk of familial Alzheimer's disease. Neuroscience Letters, 186, 17-20. doi:10.1016/0304-3940(95)11270-7

[3] Volz, H.R., Riehemann, S., Maurer, I., Smesny, S., Sommer, M., Rzanny, R., Holstein, W., Czekalla, J. and Sauer, H. (2000) Reduced phosphodiesters and highenergy phosphates in the frontal lobe of schizophrenic patients: A ${ }^{31} \mathrm{P}$ chemical shift spectroscopic-imaging study. Biological Psychiatry, 47, 954-961. doi:10.1016/S0006-3223(00)00235-3

[4] Maurer, I., Zierz, S. and Moller, H.-J. (2001) Evidence for a mitochondrial oxidative phosphorylation defect in brains from patients with schizophrenia. Schizophrenia Research, 48, 125-136.

doi:10.1016/S0920-9964(00)00075-X

[5] Fukuzako, H. (2001) Neurochemical investigation of the schizophrenic brain by in vivo phosphorus magnetic resonance spectroscopy. The World Journal of Biological Psychiatry, 2, 70-82. doi:10.3109/15622970109027496

[6] Jou, S.-H., Chiu, N.-Y. and Liu, C.-S. (2009) Mitochondrial dysfunction and psychiatric disorders. Chang Gung Medical Journal, 32, 370-379.

[7] Buchsbaum, M.S. and Hazlett, E.A. (1998) Positron emission tomography studies of abnormal glucose metabolism in schizophrenia. Schizophrenia Bulletin, 24, 343-364.

[8] Cavelier, L., Jazin, E.E., Eriksson, I., Prince, J., Bave, U., Oreland, L. and Gyllensten, U. (1995) Decreased cytochrome c-oxidase activity and lack of age-related accumulation of mitochondrial DNA deletions in the brains of schizophrenics. Genomics, 29, 217-224.

doi:10.1006/geno.1995.1234

[9] Shao, L., Martin, M.V., Watson, S.J., Schatzberg, A., Akil, H., Myers, R.M., Jones, E.G., Bunney, W.E. and Vawter, M.P. (2008) Mitochondrial involvement in psychiatric disorders. Annals of Medicine, 40, 281-295.

[10] Mann, V.M., Cooper, J.M., Krige, D., Daniel, S.E., Schapira, A.H.V. and Marsden, C.D. (1992) Brain, skeletal muscle and platelet homogenate mitochondrial function in Parkinson's disease. Brain, 115, 333-342.

[11] Dror, N., Klein, E., Karry, R., Sheinkman, A., Kirsh, Z., Mazor, M., Tzukerman, M. and Ben-Shachar D. (2002) State-dependent alterations in mitochondrial complex I activity in platelets: A potential peripheral marker for schizophrenia. Molecular Psychiatry, 7, 995-1001.

[12] Böhm, M., Papezova, H., Hansikova, H., Wenchich, L. and Zeman, J. (2007) Activities of respiratory chain complexes in isolated platelets in females with anorexia nervosa. International Journal of Eating Disorders, 40, 659-663. doi:10.1002/eat.20403

[13] Ben-Shachar, D. and Klein, E. (2008) Methods and kits for diagnosis of schizophrenia. US Patent, 7, 442-496.

[14] Valla, J., Schneider, L., Niedzielko, T., Coon, K.D., Caselli, R., Sabbagh, M.N., Ahern, G.L., Baxter, L., Alexander, G., Walker, D.G. and Reiman, E.M. (2006) Impaired platelet mitochondrial activity in Alzheimer's disease and mild cognitive impairment. Mitochondrion, 6, 323-330.

[15] Burbaeva, G.Sh., Boksha, I.S., Turishcheva, M.S., Tereshkina, E.B., Savushkina, O.K., Starodubtseva, L.I., Fedorova, Ya.B. and Gavrilova, S.I. (2008) Cytochrome coxidase and glutamine synthetase-like protein in blood platelets as candidates for the role of early markers of Alzheimer's disease. Proceedings of the IV-th Scientific and Practical Conference "Alzheimer's disease and cognitive impairments in old age: Advances in neurobiology and therapy”, 89-97 [Russian].

[16] Boksha, I., Burbaeva, G., Savushkina, O., Tereshkina, E., Turishcheva, M., Starodubtseva, L. and Vorobyeva, E. (2009) Blood proteins as markers and predictors of cognitive deficit in mental pathologies. Abstracts, 3rd World Congress Gene-2009, Foshan, China.

http://www.bitlifesciences.com/wcg2009/Program.asp

[17] Benecke, R., Strümper, P. and Weiss, H. (1993) Electron transfer complexes I and IV of platelets are abnormal in Parkinson's disease but normal in Parkinson-plus syndromes. Brain 116, 1451-1463.

doi:10.1093/brain/116.6.1451

[18] Parker, W.D. Jr and Parks, J.K. (1995) Cytochrome coxidase in Alzheimer's disease brain: purification and characterization. Neurology, 45, 482-486.

[19] Cardoso, S.M., Proenca, M.T., Santos, S., Santana, I. and Oliveira, C.R. (2004) Cytochrome c-oxidase is decreased in Alzheimer's disease platelets. Neurobiology of Aging, 25, 105-110. doi:10.1016/S0197-4580(03)00033-2

[20] Lowry, O.H., Rosebrough, N.J., Farr, A.L. and Randall, R.J. (1951) Protein measurement with the folin phenol reagent. The Journal of Biological Chemistry, 193, 265275.

[21] Kato, T. (2001) The other, forgotten genome: Mitochondrial DNA and mental disorders. Molecular Psychiatry, 6, 625-633. doi:10.1038/sj.mp.4000926

[22] Washizuka, S., Iwamoto, K., Kakiuchi, C., Bundo, M. and Kato, T. (2009) Expression of mitochondrial complex I subunit gene NDUFV2 in the lymphoblastoid cells derived from patients with bipolar disorder and schizophrenia. Neuroscience Research, 63, 199-204. doi:10.1016/j.neures.2008.12.004

[23] Maurer, I. and Moller, H.J. (1997) Inhibition of complex I by neuroleptics in normal human brain cortex parallels the extrapyramidal toxicity of neuroleptics. Molecular and Cellular Biochemistry, 174, 255-259. doi:10.1023/A:1006872911332

[24] Streck, E.L., Rezin, G.T., Barbosa, L.M., Assis, L.C., Grandi, E. and Quevedo, J. (2007) Effect of antipsychotics on succinate dehydrogenase and cytochrome oxidase activities in rat brain. Naunyn-Schmiedeberg's Archives 
of Pharmacology, 376, 127-133. doi:10.1007/s00210-007-0178-2

[25] Casademont, J., Garrabou, G., Miró, Ò., López, S., Pons, A., Bernardo, M. and Cardellach, F. (2007) Neuroleptic treatment effect on mitochondrial electron transport chain: Peripheral blood mononuclear cells analysis in psychotic patients. Journal of Clinical Psychopharmacology, 27, 284-288. doi:10.1097/JCP.0b013e318054753e

[26] Burbaeva, G.Sh., Boksha, I.S., Tereshkina, E.B., Savushkina, O.K., Turishcheva, M.S., Starodubtseva, L.I., Brusov, O.S. and Morozova, M.A. (2006) Effect of olanzapine treatment on platelet glutamine synthetase-like pro- tein and glutamate dehydrogenase immunoreactivity in schizophrenia. The World Journal of Biological Psychiatry, 7, 75-81. doi:10.1080/15622970510029957

[27] Wechsler, D. (1981) Wechsler adult intelligence scalerevised,San Antonio, TX: Psychological Corporation.

[28] Marder, S.R., Davis, J.M. and Chouinard, G. (1997) The effects of risperidone on the five dimensions of schizophrenia derived by factor analysis: combined results of the North American trials. The Journal of Clinical Psychiatry, 58, 538-546. doi:10.4088/JCP.v58n1205 